\title{
$N K_{0}$ and $N K_{1}$ of the groups $C_{4}$ and $D_{4}$
}

\author{
Addendum to "Lower algebraic $K$-theory of hyperbolic 3-simplex reflection \\ groups" by J.-F. Lafont and I. J. Ortiz
}

Charles Weibel

\begin{abstract}
In this addendum to [LO] we explicitly compute the Bass Nil-groups $N K_{i}\left(\mathbb{Z}\left[C_{4}\right]\right)$ for $i=0,1$ and $N K_{0}\left(\mathbb{Z}\left[D_{4}\right]\right)$. We also show that $N K_{1}\left(\mathbb{Z}\left[D_{4}\right]\right)$ is not trivial. Here $C_{4}$ denotes the cyclic group of order 4 and $D_{4}$ is the dihedral group of order 8 .
\end{abstract}

Mathematics Subject Classification (2000). 19A31, 19B28, 19D35, 18F25, 16E20.

Keywords. Lower algebraic $K$-theory, hyperbolic reflection group, Bass Nil-groups.

In [LO], Lafont and Ortiz computed the lower algebraic $K$-theory of the integral group ring of all 32 hyperbolic 3-simplex reflection groups (see [LO, Tables 6-7]). For 25 of these integral group rings, their computation was completely explicit. For the remaining 7 examples, the expression for some of the $K$-groups involved the Bass Nil-groups $N K_{0}$ and $N K_{1}$ associated to $D_{4}$ (the dihedral group of order 8).

In [L05], Lück computed the lower algebraic $K$-theory of the integral group ring of the semi-direct product of the three-dimensional discrete Heisenberg group by $C_{4}$ (the cyclic group of order 4). These computations involved the Bass Nil-groups $N K_{0}$ and $N K_{1}$ associated to $C_{4}$ (see [L05, Corollary 3.9]).

In this addendum we compute the Bass Nil-groups

$$
N K_{n}(\mathbb{Z} G)=\operatorname{ker}\left\{K_{n}(\mathbb{Z} G[x]) \stackrel{x \mapsto 0}{\longrightarrow} K_{n}(\mathbb{Z} G)\right\},
$$

where $G$ is $D_{2}, C_{4}$ or $D_{4}$, and $n=0,1$. We will use these calculations to complement the calculations of [L05] and [LO] in 1.5 and 2.9 below.

Our calculation will keep track of the additional structure on the groups $N K_{n}(A)$ given by the Verschiebung and Frobenius operators, $V_{m}$ and $F_{m}$, as well as the continuous module structure over the ring $W(\mathbb{Z})$ of big Witt vectors; the additive group of $W(\mathbb{Z})$ is the abelian group $(1+x \mathbb{Z}[[x]])^{\times}$. (See [We80] for more details.) In fact, it is a module over the slightly larger Cartier algebra consisting of row-and-column 
finite sums $\sum V_{m}\left[a_{m n}\right] F_{n}$, where $V_{m}$ and $F_{m}$ are the Verschiebung and Frobenius operators, and the $[a]$ are the homotheties operators for $a \in \mathbb{Z}$; see [DW93] as well as Remarks 1.2.1 and 2.4 below. Some of the identities satisfied by these operators include: $V_{m} V_{n}=V_{m n}, F_{m} F_{n}=F_{m n}, F_{m} V_{m}=m,[a] V_{m}=V_{m}\left[a^{m}\right]$ and $F_{m}[a]=\left[a^{m}\right] F_{m}$.

It is convenient to write $V$ for the continuous $W\left(\mathbb{F}_{2}\right)$-module $x \mathbb{F}_{2}[x]$, which, as an abelian group, is just a countable direct sum of copies of $\mathbb{F}_{2}=\mathbb{Z} / 2$ on generators $x^{i}$, $i>0$. The module structure on $V$ is determined by: $V_{m}\left(x^{n}\right)=x^{m n} ;[a] x^{n}=a^{n} x^{n}$; $F_{m}\left(x^{n}\right)=0$ if $(m, n)=1(m>1)$ and $F_{d}\left(x^{n}\right)=d x^{n / d}$ when $d \mid n$.

\section{The groups $C_{2}, D_{2}$ and $C_{4}$}

For the cyclic group $C_{2}=\langle\sigma\rangle$ of order two, consider the Rim square:

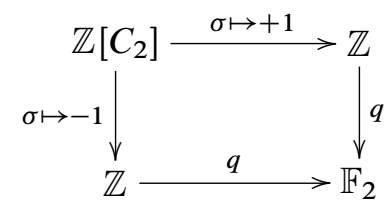

or, equivalently,

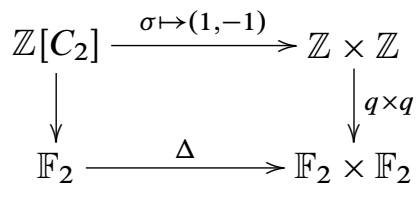

from which we immediately get $N K_{0}\left(\mathbb{Z}\left[C_{2}\right]\right)=N K_{1}\left(\mathbb{Z}\left[C_{2}\right]\right)=0$ as in [Bas68, XII.10.6] and [Mi71, 6.4]. From Guin-Loday-Keune [GL80], [Keu81], the double relative group $N K_{2}\left(\mathbb{Z}\left[C_{2}\right], \sigma+1, \sigma-1\right)$ is isomorphic to $V$, with the Dennis-Stein symbol $\left\langle x^{n}(\sigma-1), \sigma+1\right\rangle$ corresponding to $x^{n} \in V$. We also have a diagram

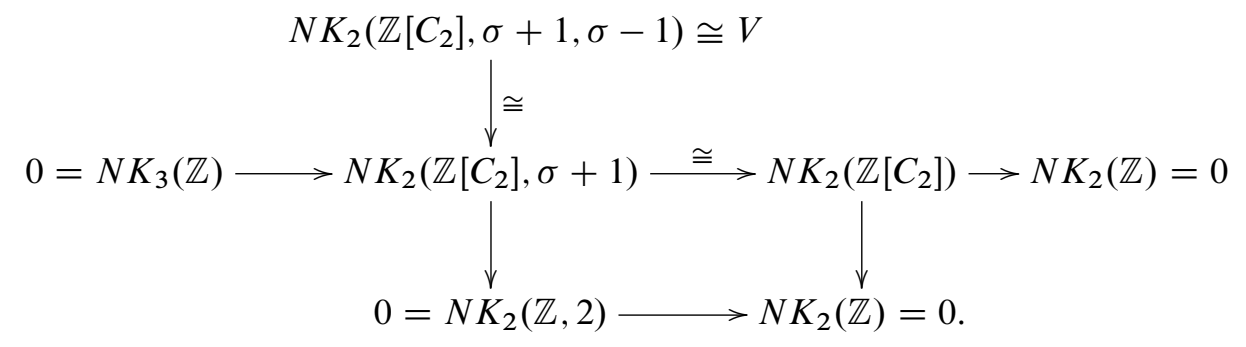

Thus we obtain:

Theorem 1.1. $N K_{2}\left(\mathbb{Z}\left[C_{2}\right]\right) \cong V$ with $\left\langle x^{n}(\sigma-1), \sigma+1\right\rangle$ corresponding to $x^{n} \in V$.

We now turn to the group $D_{2}=C_{2} \times C_{2}$. First we need a calculation. Let $\Phi(V)$ denote the subgroup (and Cartier submodule) $x^{2} \mathbb{F}_{2}\left[x^{2}\right]$ of $V$, and write $\Omega_{R}$ for the Kähler differentials of $R$, so that $\Omega_{\mathbb{F}_{2}[x]} \cong \mathbb{F}_{2}[x] d x$. By abuse, we will write $\mathbb{F}_{2}[\varepsilon]$ for the 2-dimensional algebra $\mathbb{F}_{2}[\varepsilon] /\left(\varepsilon^{2}\right)$. 
Lemma 1.2. The map $q: \mathbb{Z}\left[C_{2}\right] \rightarrow \mathbb{F}_{2}\left[C_{2}\right] \cong \mathbb{F}_{2}[\varepsilon]$ in (1) induces an exact sequence

$$
0 \longrightarrow \Phi(V) \longrightarrow N K_{2}\left(\mathbb{Z}\left[C_{2}\right]\right) \stackrel{q}{\longrightarrow} N K_{2}\left(\mathbb{F}_{2}[\varepsilon]\right) \stackrel{D}{\longrightarrow} \Omega_{\mathbb{F}_{2}[x]} \longrightarrow 0 .
$$

Proof. Van der Kallen computed $N_{2}\left(\mathbb{F}_{2}[\varepsilon]\right)$ in [vdK71, Exemple 3]: there is a split short exact sequence

$$
0 \longrightarrow V / \Phi(V) \stackrel{F}{\rightarrow} N K_{2}\left(\mathbb{F}_{2}[\varepsilon]\right) \stackrel{D}{\rightarrow} \Omega_{\mathbb{F}_{2}[x]} \longrightarrow 0,
$$

where $F\left(x^{n}\right)=\left\langle x^{n} \varepsilon, \varepsilon\right\rangle$ and $D\left(\left\langle f \varepsilon, g+g^{\prime} \varepsilon\right\rangle\right)=f d g$. The map $N K_{2}\left(\mathbb{Z}\left[C_{2}\right]\right) \stackrel{q}{\rightarrow}$ $N K_{2}\left(\mathbb{F}_{2}[\varepsilon]\right)$ sends $\left\langle x^{n}(\sigma+1), \sigma-1\right\rangle$ to $F\left(x^{n}\right)=\left\langle x^{n} \varepsilon, \varepsilon\right\rangle$. By Theorem 1.1 and (2), this map has kernel $\Phi(V)$ and image $F(V / \Phi(V))$.

Remark 1.2.1. Although $\Omega_{\mathbb{F}_{2}[x]}$ is isomorphic to $V$ as an abelian group, it has a different $W\left(\mathbb{F}_{2}\right)$-module structure. This is determined by the formulas in $\Omega_{\mathbb{F}_{2}[x]}$ :

$$
V_{m}\left(x^{n-1} d x\right)=m x^{m n-1} d x, \quad F_{m}\left(x^{n-1} d x\right)= \begin{cases}x^{n / m-1} d x & \text { if } m \mid n, \\ 0 & \text { else. }\end{cases}
$$

Grunewald has pointed out that since $\Omega_{\mathbb{F}_{2}[x]}$ is not finitely generated as a module over the $\mathbb{F}_{2}$-Cartier algebra (of row-and-column finite sums $\sum V_{m}\left[a_{m n}\right] F_{n}$ ), or over the subalgebra $\left.W\left(\mathbb{F}_{2}\right)\right)$, neither are $N K_{2}\left(\mathbb{F}_{2}[\varepsilon]\right)$ or (by 1.3 below) $N K_{1}\left(\mathbb{Z}\left[D_{2}\right]\right)$.

Theorem 1.3. For $D_{2}=C_{2} \times C_{2}, N K_{0}\left(\mathbb{Z}\left[D_{2}\right]\right) \cong V$, $N K_{1}\left(\mathbb{Z}\left[D_{2}\right]\right) \cong \Omega_{\mathbb{F}_{2}[x]}$ and the image of the map $N K_{2}\left(\mathbb{Z}\left[D_{2}\right]\right) \rightarrow N K_{2}\left(\mathbb{Z}\left[C_{2}\right]\right)^{2} \cong V^{2}$ is $\Phi(V) \times V$.

Proof. We tensor (1) with $\mathbb{Z}\left[C_{2}\right]$. Since $\mathbb{F}_{2}\left[C_{2}\right] \cong \mathbb{F}_{2}[\varepsilon], \varepsilon^{2}=0$, and $N K_{1}\left(\mathbb{F}_{2}\left[C_{2}\right]\right) \cong$ $\left(1+x \varepsilon \mathbb{F}_{2}[x]\right)^{\times} \cong V$, then the Mayer-Vietoris sequence in [Mi71, Theorem 6.4] for the $N K$-functor,

$$
\begin{aligned}
& N K_{2}\left(\mathbb{Z}\left[D_{2}\right]\right) \longrightarrow\left(N K_{2}\left(\mathbb{Z}\left[C_{2}\right]\right)\right)^{2} \stackrel{q \times q}{\longrightarrow} N K_{2}\left(\mathbb{F}_{2}[\varepsilon]\right) \longrightarrow N K_{1}\left(\mathbb{Z}\left[D_{2}\right]\right) \\
& \longrightarrow\left(N K_{1}\left(\mathbb{Z}\left[C_{2}\right]\right)^{2} \longrightarrow N K_{1}\left(\mathbb{F}_{2}[\varepsilon]\right) \stackrel{\cong}{\longrightarrow} N K_{0}\left(\mathbb{Z}\left[D_{2}\right]\right) \longrightarrow N K_{0}\left(\mathbb{Z}\left[C_{2}\right]\right),\right.
\end{aligned}
$$

quickly gives $N K_{0}\left(\mathbb{Z}\left[D_{2}\right]\right) \cong N K_{1}\left(\mathbb{F}_{2}[\varepsilon]\right) \cong V$. By Lemma 1.2, the initial portion of (3) yields the calculation of $N K_{1}\left(\mathbb{Z}\left[D_{2}\right]\right)$ and the asserted surjection $N K_{2}\left(\mathbb{Z}\left[D_{2}\right]\right) \rightarrow \Phi(V) \times V$.

Remark. The kernel $K$ of the map $N K_{2}\left(\mathbb{Z}\left[D_{2}\right]\right) \rightarrow V^{2}$ in Theorem 1.3 has a subgroup generated by the double relative group $N K_{2}\left(\mathbb{Z}\left[D_{2}\right], \sigma_{1}+1, \sigma_{1}-1\right)$, which is isomorphic to $\mathbb{F}_{2}[\varepsilon] \otimes V$ on the symbols $\left\langle x^{n}\left(a+b \sigma_{2}\right)\left(\sigma_{1}+1\right), \sigma_{2}-1\right\rangle$, where $\sigma_{1}, \sigma_{2}$ are the generators of $D_{2}=C_{2} \times C_{2}$. The quotient of $K$ by this subgroup is generated by the image of $N K_{3}\left(\mathbb{F}_{2}[\varepsilon]\right)$, a group which I do not know. 
The analysis for the cyclic group $C_{4}$ of order 4 on generator $\sigma$ is similar, using the Rim square

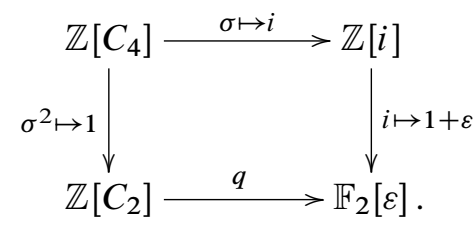

Theorem 1.4. $N K_{1}\left(\mathbb{Z}\left[C_{4}\right]\right) \cong \Omega_{F_{2}[x]}$ and $N K_{0}\left(\mathbb{Z}\left[C_{4}\right]\right) \cong V$.

Proof. Since $\mathbb{Z}[i]$ is a regular ring, the Mayer-Vietoris sequence for (4) reduces to

$$
\begin{aligned}
& N K_{2}\left(\mathbb{Z}\left[C_{4}\right]\right) \stackrel{p_{2}}{\longrightarrow} N K_{2}\left(\mathbb{Z}\left[C_{2}\right]\right) \stackrel{q}{\longrightarrow} N K_{2}\left(\mathbb{F}_{2}[\varepsilon]\right) \longrightarrow N K_{1}\left(\mathbb{Z}\left[C_{4}\right]\right) \\
& \quad \longrightarrow N K_{1}\left(\mathbb{Z}\left[C_{2}\right]\right) \rightarrow N K_{1}\left(\mathbb{F}_{2}[\varepsilon]\right) \stackrel{\cong}{\longrightarrow} N K_{0}\left(\mathbb{Z}\left[C_{4}\right]\right) \longrightarrow N K_{0}\left(\mathbb{Z}\left[C_{2}\right]\right) .
\end{aligned}
$$

The isomorphism marked in this sequence follows from Theorem 1.1. By Lemma 1.2, the image of the first map $p_{2}$ is $\Phi(V)$ and the cokernel of the map $q$ is $\Omega_{\mathbb{F}_{2}[\varepsilon] \text {. }}$.

Remark. The proof provides a surjection $N K_{2}\left(\mathbb{Z}\left[C_{4}\right]\right) \stackrel{p_{2}}{\longrightarrow} \Phi(V)$. The kernel of $p_{2}$ contains the image $E$ of the double relative group $N K_{2}\left(\mathbb{Z}\left[C_{4}\right], \sigma^{2}+1, \sigma^{2}-1\right)$, which is isomorphic to $\mathbb{F}_{2}[\varepsilon] \otimes V$ on symbols $\left\langle\sigma^{2}+1, x^{n}\left(\sigma^{2}-1\right)\right\rangle$. The quotient $\operatorname{ker}\left(p_{2}\right) / E$ is generated by the image of $N K_{3}\left(\mathbb{F}_{2}[\varepsilon]\right)$, which I do not know.

Here is an application of this calculation. Let Hei denote the three-dimensional discrete Heisenberg group, which is the subgroup of $G L(3, \mathbb{Z})$ consisting of upper triangular integral matrices with ones along the diagonal. Consider the action of the cyclic group $C_{4}$ given by

$$
\left(\begin{array}{ccc}
1 & x & y \\
0 & 1 & z \\
0 & 0 & 1
\end{array}\right) \mapsto\left(\begin{array}{ccc}
1 & -z & y-x z \\
0 & 1 & x \\
0 & 0 & 1
\end{array}\right) .
$$

Combining Theorem 1.4 with [L05, 3.9], the lower $K$-theory of the group Hei $\rtimes C_{4}$ is given in the following proposition.

Proposition 1.5. We have

$$
\mathrm{Wh}_{n}\left(\text { Hei } \rtimes C_{4}\right)= \begin{cases}\bigoplus_{\infty} \mathbb{Z} / 2, & n=0,1, \\ 0, & n \leq-1 .\end{cases}
$$




\section{The dihedral group $D_{4}$}

Before moving on to the group ring of $D_{4}$, we need some facts about the double relative groups $K_{1}(A, B, I)$ when $A \rightarrow B$ is an injection. These groups were described in [GW83, 0.2] as follows:

$$
K_{1}(A, B, I) \cong(B / A) \otimes\left(I / I^{2}\right) /\{b \otimes c z+c \otimes z b-b c \otimes z\} \quad(b, c \in B, z \in I) .
$$

Moreover, by [GW83, 3.12 and 4.1], the map $K_{1}(A, B, I) \rightarrow K_{1}(A, I)$ sends the class of $b \otimes z$ to the class of the matrix $\left(\begin{array}{cc}1-z b & z \\ -b z b & 1+b z\end{array}\right)$.

Lemma 2.1. Suppose that $A \rightarrow B$ is a ring homomorphism mapping an ideal I of $A$ isomorphically onto an ideal of $B$. Then the double relative group satisfies

$$
K_{1}(A[x], B[x], I[x]) \cong K_{1}(A, B, I) \otimes \mathbb{Z}[x],
$$

and

$$
N K_{1}(A, B, I) \cong K_{1}(A, B, I) \otimes x \mathbb{Z}[x]
$$

Proof. Because $x$ is central in $B[x]$, the formulas are immediate from (5).

We will be specifically interested in the twisted group ring $A=\mathbb{Z}[i] \rtimes C_{2}$, where $C_{2}=\langle\tau\rangle$ acts on $\mathbb{Z}[i]$ by $\tau i \tau^{-1}=-i$. It injects into the matrix ring $B=M_{2}(\mathbb{Z})$ by the map $\phi: A \rightarrow B$ defined by $\phi(i)=\left(\begin{array}{cc}0 & 1 \\ -1 & 0\end{array}\right)$ and $\phi(\tau)=\left(\begin{array}{cc}1 & 0 \\ 0 & -1\end{array}\right)$. The ideal $I=(2,1+\tau) A$ maps isomorphically to $2 B$, and $A / I \cong \mathbb{F}_{2}\left[\varepsilon_{1}\right]$, where $\varepsilon_{1}=1+i$ and $\varepsilon_{1}^{2}=0$. Hence we have the following cartesian square:

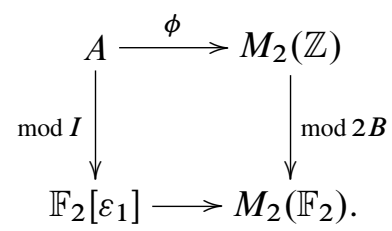

To calculate $N K_{1}(A)$, we use the following double relative calculation.

Lemma 2.2. The double relative group $K_{1}(A, B, 2 B)$ of $(6)$ is isomorphic to $\mathbb{F}_{2}$, and $N K_{1}(A, B, 2 B) \cong V$. The map $V \cong N K_{1}(A, B, 2 B) \rightarrow N K_{1}(A)$ sends $x^{n} \in V$ to the class of the unit $1+x^{n} i(1+\tau)$ of $A[x]$.

Proof. Since $\operatorname{dim}(B / A)=2$ and $\operatorname{dim}\left(I / I^{2}\right)=4$, the group $(B / A) \otimes\left(I / I^{2}\right)$ has 8 generators and 64 relations; a basis of $B / A$ is $\left\{e_{11}, e_{12}\right\}$ and the $2 e_{i j}$ span $I / I^{2}$. By inspection of the relations in (5) we see that the map $B / I \otimes I / I^{2} \rightarrow \mathbb{F}_{2}$ sending $e_{i j} \otimes 2 e_{k l}$ to $\delta_{i l}+\delta_{j k}$ sends $A / I \otimes I / I^{2}$ and all the relations in (5) to zero, and 
sends $e_{11} \otimes 2 e_{12}$ to 1 . Thus it induces a surjection $K_{1}(A, B, I) \rightarrow \mathbb{F}_{2}$. We claim that this is an isomorphism.

The relations for $(b, c, z)=\left(e_{11}, e_{11}, 2 e_{11}\right),\left(e_{11}, e_{11}, 2 e_{22}\right),\left(e_{11}, e_{12}, 2 e_{12}\right)$ and $\left(e_{11}, e_{21}, 2 e_{21}\right)$ in $(5)$ yield the relations

$$
0=e_{11} \otimes 2 e_{11}=e_{11} \otimes 2 e_{22}=e_{12} \otimes 2 e_{12}=e_{21} \otimes 2 e_{21} .
$$

The relations for $\left(e_{12}, e_{11}, 2 e_{11}\right),(b, c, z)=\left(e_{11}, e_{21}, 2 e_{11}\right)$ and $\left(e_{12}, e_{12}, 2 e_{21}\right)$ in (5) yield the relations

$$
0=e_{11} \otimes 2 e_{12}-e_{12} \otimes 2 e_{11}=e_{21} \otimes 2 e_{11}-e_{11} \otimes 2 e_{21}=e_{12} \otimes 2 e_{22}-e_{12} \otimes 2 e_{11} .
$$

This verifies the claim, proving that $K_{1}(A, B, 2 B) \cong \mathbb{F}_{2}$.

Finally, the map $N K_{1}(A, B, 2 B) \rightarrow N K_{1}(A, I)$ sends the class of $x^{n} e_{11} \otimes 2 e_{12}$ to the class of the matrix $\left(\begin{array}{cc}1-2 x^{n} e_{12} e_{11} & 2 x^{n} e_{12} \\ -x^{2 n} e_{11} e_{12} e_{11} & 1+2 x^{n} e_{11} e_{12}\end{array}\right)=\left(\begin{array}{cc}1 & x^{n} i(1+\tau) \\ 0 & 1+x^{n} i(1+\tau)\end{array}\right)$, which is the class of $1+x^{n} i(1+\tau)$ in $N K_{1}(A)$.

Remark. The elements $u=i+\tau$ and $v=i(1+\tau)$ of $A$ satisfy $u^{2}=v^{2}=0$, and are distinct in $A / 2 A=\mathbb{F}_{2}[i, \tau]$. Hence the units $\left(1+x^{m} u\right)\left(1+x^{n} v\right)$ of $A[x]$ generate a subgroup of $N K_{1}(A)$ isomorphic to $V^{2}$, which injects into $N K_{1}\left(\mathbb{F}_{2}[i, \tau]\right) \cong V^{3}$. (Since $\mathbb{F}_{2}[i, \tau]=\mathbb{F}_{2}[u, v] /\left(u^{2}, v^{2}\right)$, the other copy of $V$ in $N K_{1}\left(\mathbb{F}_{2}[i, \tau]\right)$ is the subgroup generated by all $\left(1+x^{n} u v\right)$.)

Proposition 2.3. $N K_{0}(A)=0$ and $N K_{1}(A) \cong V^{2}$ on the units $\left(1+x^{m} u\right)\left(1+x^{n} v\right)$.

The maps $A \rightarrow \mathbb{F}_{2}[i, \tau] \cong \mathbb{F}_{2}\left[\varepsilon_{1}, \varepsilon_{2}\right]$ and $\Omega_{\mathbb{F}_{2}[x]} \stackrel{\delta}{\longrightarrow} N K_{2}\left(\mathbb{F}_{2}\left[\varepsilon_{1}, \varepsilon_{2}\right]\right)$ sending $x^{n}$ to $\left\langle x^{n-1} \varepsilon_{1} \varepsilon_{2}, x\right\rangle$ induce a surjection $N K_{2}(A) \times \Omega_{\mathbb{F}_{2}[x]} \rightarrow N K_{2}\left(\mathbb{F}_{2}\left[\varepsilon_{1}, \varepsilon_{2}\right]\right)$.

Proof. Consider the Mayer-Vietoris sequence of the square (6). Since $B=M_{2}(\mathbb{Z})$ and $B / I=M_{2}\left(\mathbb{F}_{2}\right)$ are regular rings, $N K_{n}(B)=N K_{n}(B / I)=0$ and hence $N K_{n}(B, I)=0$ for all $n$. We immediately get that $N K_{n}(A, B, I) \cong N K_{n}(A, I)$, that the Mayer-Vietoris sequence reduces to $N K_{0}(A) \cong N K_{0}(A / I)=0$, and that there is an exact sequence

$$
N K_{2}(A) \rightarrow N K_{2}\left(\mathbb{F}_{2}\left[\varepsilon_{1}\right]\right) \rightarrow N K_{1}(A, B, I) \rightarrow N K_{1}(A) \rightarrow N K_{1}\left(\mathbb{F}_{2}\left[\varepsilon_{1}\right]\right) \rightarrow 0 .
$$

By Lemma 2.2 and the remark preceding it, this yields the calculation of $N K_{1}(A)$.

Now $\pi: A \rightarrow \mathbb{F}_{2}\left[\varepsilon_{1}, \varepsilon_{2}\right]$ satisfies $\pi(u)=\varepsilon_{1}+\varepsilon_{2}, \pi(v)=\varepsilon_{1}+\varepsilon_{1} \varepsilon_{2}$ and $\pi(u v)=\varepsilon_{1} \varepsilon_{2}$, so we may write $\mathbb{F}_{2}\left[\varepsilon_{1}, \varepsilon_{2}\right] \cong \mathbb{F}_{2}[\bar{u}, \bar{v}] /\left(\bar{u}^{2}, \bar{v}^{2}\right)$. By [vdK71], the group $N K_{2}\left(\mathbb{F}_{2}[\bar{u}, \bar{v}]\right)$ is isomorphic to the direct sum of $N K_{2}\left(\mathbb{F}_{2}[\bar{u}]\right), N K_{2}\left(\mathbb{F}_{2}[\bar{v}]\right)$ and a group with the following generators:

$$
\left\langle x^{n} \bar{u}, \bar{v}\right\rangle, \quad\left\langle x^{n} \bar{u} \bar{v}, \bar{u}\right\rangle, \quad\left\langle x^{n} \bar{u} \bar{v}, \bar{v}\right\rangle \quad \text { and } \quad\left\langle x^{n-1} \bar{u} \bar{v}, x\right\rangle .
$$


Since $u^{2}=v^{2}=0$ in $A$, all these symbols lift to Dennis-Stein symbols in $N K_{2}(A)$ except possibly the symbols $\left\langle x^{n-1} \bar{u} \bar{v}, x\right\rangle$. But these symbols are hit by the image of $\Omega_{\mathbb{F}_{2}[x]}$ under $\delta$.

Remark. $\delta: \Omega_{\mathbb{F}_{2}[x]} \rightarrow N K_{2}\left(\mathbb{F}_{2}\left[\varepsilon_{1}, \varepsilon_{2}\right]\right)$ is a homomorphism by the Dennis-Stein identity $\langle f, x\rangle\langle g, x\rangle=\langle f+g-f g x, x\rangle$ with $f g=0$; see [GL80, p. 184]. It is a morphism of $\mathbb{F}_{2}$-Cartier modules since $V_{m}\left\langle x^{n-1} \varepsilon_{1} \varepsilon_{2}, x\right\rangle=m\left\langle x^{m n-1} \varepsilon_{1} \varepsilon_{2}, x\right\rangle=$ $\delta\left(V_{m}\left(x^{n}\right)\right)$ and (by [St80, 2.1])

$$
\begin{aligned}
& F_{m}\left\langle x^{n-1} \varepsilon_{1} \varepsilon_{2}, x\right\rangle \\
& \quad= \begin{cases}\left\langle x^{n / m-1} \varepsilon_{1} \varepsilon_{2}, x\right\rangle, & m \mid n \\
r\left\langle x^{n-1}\left(\varepsilon_{1} \varepsilon_{2}\right)^{m}, x\right\rangle-s\left\langle x^{n}\left(\varepsilon_{1} \varepsilon_{2}\right)^{m-1}, \varepsilon_{1} \varepsilon_{2}\right\rangle=0, & r m+s n=1 .\end{cases}
\end{aligned}
$$

Our analysis of $D_{4}$ will involve the units of the ring $\mathbb{Z} / 4[x]\left[C_{2}\right]$.

Example 2.4. Consider the modular group ring $B=\mathbb{Z} / 4\left[C_{2}\right]=\mathbb{Z} / 4[e] /\left(e^{2}-2 e\right)$, withe $=1-\tau$. The ideals $2 e B$ of $B$ and $e B / 2 e B$ of $B / 2 e B$ are isomorphic to $\mathbb{F}_{2}$, so both $N K_{1}(B, 2 e)$ and $N K_{1}(B / 2 e, e)$ are isomorphic to $V$ and the group $N K_{1}(B, e)$, identified with the abelian group $(1+x e B[x])^{\times}$, is a nontrivial extension:

$$
0 \rightarrow V \rightarrow N K_{1}(B, e) \rightarrow V \rightarrow 0 .
$$

As an abelian group, $N K_{1}(B, e)$ is the direct sum of a countably infinite free $\mathbb{Z} / 4$-module on the $\left(1+\right.$ ex $\left.x^{m}\right)(m=1,2, \ldots)$ and a countably infinite free $\mathbb{Z} / 2$ module on the $\left(1+2 e x^{2 i-1}\right)(i=1,2, \ldots)$. As a module over the $\mathbb{Z} / 4$-Cartier algebra (generated by the operators $V_{m}, F_{m}$ and homothety [2]), $N K_{1}(B, e)$ is cyclic on generator $u=1+e x ; V_{m}(u)=1+e x^{m}$ and $V_{m}[2](u)=1+2 e x^{m}$.

Finally, we are in position to analyze $N K_{0}\left(\mathbb{Z}\left[D_{4}\right]\right)$. The sharp exponent 4 for $N K_{0}\left(\mathbb{Z}\left[D_{4}\right]\right)$ in Theorem 2.5 is a slight improvement on the bound in [CP02]. It is convenient to write $D_{4}$ as the semidirect product of $C_{4}$ (on $\sigma$ ) with the cyclic group $C_{2}=\{1, \tau\}$, with relation $\tau \sigma \tau=\sigma^{-1}$.

Theorem 2.5. The group $N K_{0}\left(\mathbb{Z}\left[D_{4}\right]\right)$ is isomorphic to the cyclic Cartier module $N K_{1}\left(\mathbb{Z} / 4\left[C_{2}\right], 1-\tau\right)$, described in Example 2.4. As a group, it is the direct sum of a countably infinite free $\mathbb{Z} / 4$-module and a countably infinite free $\mathbb{Z} / 2$-module.

Proof. We can map $\mathbb{Z}\left[D_{4}\right]$ to the twisted ring $A=\mathbb{Z}[i] \rtimes C_{2}$ occurring in (6) above, sending $\sigma$ to $i$. Combining this with the natural surjection onto the subring $\mathbb{Z}\left[D_{2}\right]$ of $\mathbb{Z}\left[C_{2}\right] \times \mathbb{Z}\left[C_{2}\right]$, we get a ring map $\mathbb{Z}\left[D_{4}\right] \rightarrow A \times \mathbb{Z}\left[C_{2}\right] \times \mathbb{Z}\left[C_{2}\right]$. The ideal $I=\left(4,2-2 \sigma, \sigma^{2}-1\right) \mathbb{Z}\left[D_{4}\right]$ has $B_{0}=\mathbb{Z}\left[D_{4}\right] / I=\mathbb{Z} / 4\left[D_{2}\right] /(2-2 \sigma)$, and is 
isomorphic to the ideal $2 A \times(4) \times(4)$ of $A \times \mathbb{Z}\left[C_{2}\right] \times \mathbb{Z}\left[C_{2}\right]$. Consider the following cartesian square:

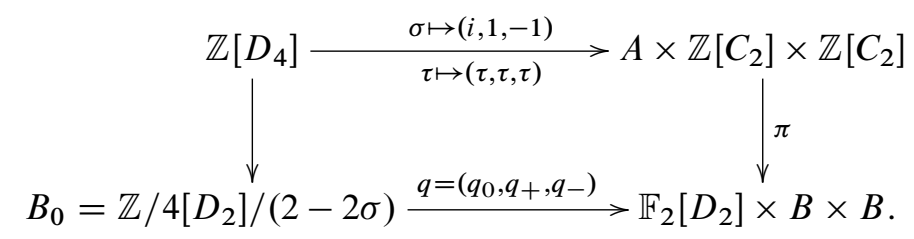

The kernel of the split surjection $q_{+}: B_{0} \rightarrow B=\mathbb{Z} / 4\left[C_{2}\right]$ is the 2-dimensional ideal $J=(1-\sigma) B_{0}$. This implies that $N K_{1}\left(B_{0}\right)=N K_{1}(B) \oplus N K_{1}\left(B_{0}, J\right)$. Because $N K_{1}\left(\mathbb{Z}\left[C_{2}\right]\right)=N K_{0}\left(\mathbb{Z}\left[C_{2}\right]\right)=N K_{0}(A)=0$ (by 2.3), the Mayer-Vietoris sequence associated to $(7)$ ends

$$
N K_{1}(A) \times N K_{1}\left(B_{0}, J\right) \stackrel{\eta}{\rightarrow} N K_{1}\left(\mathbb{F}_{2}\left[D_{2}\right]\right) \times N K_{1}(B) \rightarrow N K_{0}\left(\mathbb{Z}\left[D_{4}\right]\right) \rightarrow 0 .
$$

The displayed map $\eta$ is given by the matrix $\left(\begin{array}{cc}\pi & 0 \\ q_{0} & q_{-}\end{array}\right)$. It is easy to see that $N K_{1}\left(B_{0}, J\right)$ is isomorphic to $V^{2}$ on the terms $\left(1+(1-\sigma) x^{m}\right)$ and $\left(1+(1-\sigma) \tau x^{n}\right)$. An elementary calculation using the isomorphism $N K_{1}(A) \cong V^{2}$ of 2.3 shows that $\eta$ is an injection, sending the module $N K_{1}(A) \times N K_{1}\left(B_{0}, J\right) \cong V^{4}$ isomorphically onto the subgroup $N K_{1}\left(\mathbb{F}_{2}\left[D_{2}\right]\right) \times N K_{1}(\mathbb{Z} / 4)$. Since $N K_{1}(B)=N K_{1}(\mathbb{Z} / 4) \oplus$ $N K_{1}(B, e B), e=1-\tau$, it follows that the induced map $N K_{1}(B, e) \rightarrow N K_{0}\left(\mathbb{Z}\left[D_{4}\right]\right)$ is an isomorphism.

To begin the calculation of $N K_{1}\left(\mathbb{Z}\left[D_{4}\right]\right)$, we extend the Mayer-Vietoris sequence (8) associated to (7) to the left. This is possible by the following observation: since $B_{0}$ maps onto each of the three ring factors on the lower right of (7), the presentation (5) shows that the double relative $K_{1}$ obstruction vanishes. Because $\eta$ is an injection in (8), the continuation of the Mayer-Vietoris sequence yields the exact sequence

$$
(*) \stackrel{\left(\begin{array}{cc}
\pi & 0 \\
q_{0} & q_{-}
\end{array}\right)}{\longrightarrow} N K_{2}\left(\mathbb{F}_{2}\left[D_{2}\right]\right) \times N K_{2}(B) \rightarrow N K_{1}\left(\mathbb{Z}\left[D_{4}\right]\right) \rightarrow 0,
$$

where $(*)$ denotes $N K_{2}(A) \times N K_{2}\left(\mathbb{Z}\left[C_{2}\right]\right)^{2} \times N K_{2}\left(B_{0}, J\right)$.

Definition 2.6. The map $\cup[x]: N K_{0}\left(\mathbb{Z}\left[D_{4}\right]\right) \rightarrow N K_{1}\left(\mathbb{Z}\left[D_{4}\right]\right)$ is obtained by composing the isomorphism $N K_{1}(B, e) \cong N K_{0}\left(\mathbb{Z}\left[D_{4}\right]\right)$ of Theorem 2.5 with the canonical map $N K_{2}(B, e) \rightarrow N K_{2}(B) \rightarrow N K_{1}\left(\mathbb{Z}\left[D_{4}\right]\right)$ of (9).

Remark. There is also a canonical map $N K_{1}(B, e) \rightarrow N K_{2}(B, e)$ sending the unit $1-$ aex to $\langle a e, x\rangle$; the composition with $N K_{2}(B, e) \subset K_{2}\left(B\left[x, x^{-1}\right], e\right)$ is given by $1-$ ae $x \mapsto\{1-$ aex, $x\}$ (multiplication by the class of $x$ in $K_{1}\left(\mathbb{Z}\left[x, x^{-1}\right]\right.$ )). 
The analogous maps from $V \cong N K_{1}(B, 2 e)$ and $V \cong N K_{1}(B / 2 e B, e)$ to $\Omega_{\mathbb{F}_{2}[x]} \cong N K_{2}(B, 2 e)$ and $\Omega_{\mathbb{F}_{2}[x]} \cong N K_{2}(B / 2 e B, e)$ are compatible with the divided power map $[d]: V \rightarrow \Omega_{\mathbb{F}_{2}[x]}$ sending $x^{n}$ to $x^{n-1} d x$. Note that $[d]$ is an isomorphism of abelian groups but is not a morphism of $\mathbb{F}_{2}$-Cartier modules.

Theorem 2.7. The map $\cup[x]: N K_{0}\left(\mathbb{Z}\left[D_{4}\right]\right) \rightarrow N K_{1}\left(\mathbb{Z}\left[D_{4}\right]\right)$ in Definition 2.6 is a surjection. Hence the group $N K_{1}\left(\mathbb{Z}\left[D_{4}\right]\right)$ has exponent 2 or 4 , and there is a commutative diagram whose rows are exact:

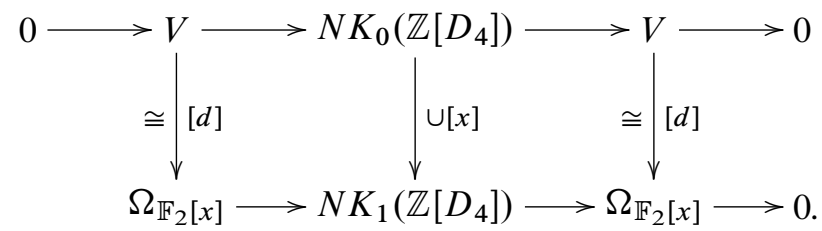

Proof. A diagram chase on (9) shows that $N K_{1}\left(\mathbb{Z}\left[D_{4}\right]\right)$ is an extension of the cokernel of $N K_{2}\left(\mathbb{Z}\left[C_{2}\right]\right) \times N K_{2}\left(B_{0}, J\right) \rightarrow N K_{2}(B)$ by a quotient of the cokernel of $N K_{2}(A) \rightarrow N K_{2}\left(\mathbb{F}_{2}\left[D_{2}\right]\right)$. These cokernels are both $\Omega_{\mathbb{F}_{2}[x]}$, by Proposition 2.3 and Lemma 2.8 below, yielding the bottom row of the theorem. The map $\cup[x]$ sends the element corresponding to $1-x^{n} a e \in N K_{1}(B, e)$ to the element corresponding to $\left\langle x^{n-1} a e, x\right\rangle \in N K_{2}(B, e)$, so the diagram in the theorem commutes by inspection.

Lemma 2.8. The cokernel of the map $N K_{2}\left(\mathbb{Z}\left[C_{2}\right]\right) \times N K_{2}\left(B_{0}, J\right) \rightarrow N K_{2}(B)$ in (9) is $\Omega_{\mathbb{F}_{2}[x]}$, on symbols $\left\langle x^{n-1} e, x\right\rangle$.

Proof. The kernel of the map $q_{-}: B_{0} \rightarrow B$ is the ideal $J^{\prime}=(1+\sigma) B_{0}$. Because $J \cap$ $J^{\prime}=0$ in $B_{0}$, the double relative group $N K_{2}\left(B_{0}, J, J^{\prime}\right)$ is isomorphic to $\mathbb{F}_{2}\left[C_{2}\right][x]$ on symbols $\left\langle x^{m}(1+\sigma),(1-\sigma)\right\rangle$ and $\left\langle x^{m} \tau(1+\sigma),(1-\sigma)\right\rangle$ by [GL80], [Keu81]. Since $J \cong 2 B$, we have an exact sequence

$$
\mathbb{F}_{2}\left[C_{2}\right][x] \rightarrow N K_{2}\left(B_{0}, J\right) \stackrel{q_{-}}{\longrightarrow} N K_{2}(B, 2 B) \rightarrow 0 .
$$

Combining this with the ideal sequence for $2 B \subset B$ shows that the cokernel of $N K_{2}\left(B_{0}, J\right) \rightarrow N K_{2}(B)$ is $N K_{2}(B / 2 B)$. Since $B / 2 B \cong \mathbb{F}_{2}\left[C_{2}\right]$, the lemma now follows from Lemma 1.2.

Inserting the calculations of Theorems 2.5 and 2.7 into Tables 6-7 in [LO], we obtain the following result.

Theorem 2.9. Let $\Gamma$ be one of the following hyperbolic 3-simplex reflection groups: $[(3,4,3,6)],\left[4,3^{[3]}\right],[4,3,6],\left[\left(3^{3}, 4\right)\right],[4,3,5],\left[(3,4)^{[2]}\right],[(3,4,3,5)]$. Then the lower algebraic $K$-theory of the groups $\Gamma$ is given by the following table: 


\begin{tabular}{|c|c|c|c|}
\hline$\Gamma$ & $K_{-1} \neq 0$ & $\tilde{K}_{0} \neq 0$ & $\mathrm{Wh} \neq 0$ \\
\hline$[(3,4,3,6)]$ & $\mathbb{Z}^{3}$ & $(\mathbb{Z} / 4)^{2} \oplus \mathrm{Nil}_{0}$ & $\mathrm{Nil}_{1}$ \\
\hline$\left[4,3^{[3]}\right]$ & $\mathbb{Z}^{3}$ & $(\mathbb{Z} / 4)^{2} \oplus \mathrm{Nil}_{0} \oplus \oplus_{\infty} \mathbb{Z} / 2$ & $\mathrm{Nil}_{1} \oplus \oplus_{\infty} \mathbb{Z} / 2$ \\
\hline$[4,3,6]$ & $\mathbb{Z}^{4}$ & $(\mathbb{Z} / 4)^{2} \oplus \mathrm{Nil}_{0} \oplus \oplus_{\infty} \mathbb{Z} / 2$ & $\mathrm{Nil}_{1} \oplus \oplus_{\infty} \mathbb{Z} / 2$ \\
\hline$\left[\left(3^{3}, 4\right)\right]$ & $\mathbb{Z}^{2}$ & $(\mathbb{Z} / 4)^{2} \oplus \mathrm{Nil}_{0} \oplus \bigoplus_{\infty} \mathbb{Z} / 2$ & $\mathrm{Nil}_{1} \oplus \oplus_{\infty} \mathbb{Z} / 2$ \\
\hline$[4,3,5]$ & $\mathbb{Z}^{4}$ & $(\mathbb{Z} / 4)^{2} \oplus \mathrm{Nil}_{0} \oplus \bigoplus_{\infty} \mathbb{Z} / 2$ & $\mathbb{Z}^{3} \oplus \mathrm{Nil}_{1} \oplus \bigoplus_{\infty} \mathbb{Z} / 2$ \\
\hline$\left[(3,4)^{[2]}\right]$ & $\mathbb{Z}^{4}$ & $(\mathbb{Z} / 4)^{4} \oplus \mathrm{Nil}_{0} \oplus \bigoplus_{\infty} \mathbb{Z} / 2$ & $2 \mathrm{Nil}_{1} \oplus \oplus_{\infty} \mathbb{Z} / 2$ \\
\hline$[(3,4,3,5)]$ & $\mathbb{Z}^{6}$ & $(\mathbb{Z} / 4)^{2} \oplus \mathrm{Nil}_{0} \oplus \bigoplus_{\infty} \mathbb{Z} / 2$ & $\mathbb{Z}^{3} \oplus \mathrm{Nil}_{1} \oplus \bigoplus_{\infty} \mathbb{Z} / 2$ \\
\hline
\end{tabular}

In this table, $\mathrm{Nil}_{0}=N K_{0}\left(\mathbb{Z}\left[D_{4}\right]\right)$ is the direct sum of a countably infinite free $\mathbb{Z} / 4$-module and a countably infinite free $\mathbb{Z} / 2$-module, and $\mathrm{Nil}_{1}=N K_{1}\left(\mathbb{Z}\left[D_{4}\right]\right)$ is a countably infinite torsion group of exponent 2 or 4 .

\section{References}

[Bas68] H. Bass, Algebraic K-theory. W. A. Benjamin, New York 1968. Zbl 0174.30302 MR 0249491

[CP02] F. Connolly and S. Parasidis, On the exponent of the cokernel of the forget-control map on $K_{0}$-groups. Fund. Math. 172 (2002), 201-216. Zbl 0992.57022 MR 1898685

[DW93] B. Dayton and C. Weibel, Module structures on the Hochschild and Cyclic Homology of Graded Rings. In Algebraic K-theory and Algebraic Topology, NATO Adv. Sci. Inst. Ser. C Math. Phys. Sci. 407, Kluwer, Dordrecht 1993, 63-90. Zbl 0900.16015 MR 1367293

[F87] F. T. Farrell, A remark on $K_{0}$ of crystallographic groups. Topology Appl. 26 (1987), 97-99. Zbl 0624.18004 MR 0893807

[GW83] S. Geller and C. Weibel, $K_{1}(A, B, I)$. J. Reine Angew. Math. 342 (1983), 12-34. Zbl 0503.18009 MR 0703484

[GL80] D. Guin-Waléry, and J-L. Loday, Obstruction à l'excision en $K$-théorie algébrique. In Algebraic K-theory (Evanston 1980), Lecture Notes in Math. 854, Springer-Verlag, Berlin 1981, 179-216. Zbl 0461.18007 MR 0618305

[Keu81] F. Keune, Doubly relative $K$-theory and the relative $K_{3}$. J. Pure Appl. Algebra 20 (1981), 39-53. Zbl 0473.18009 MR 0596152

[LO] J. F. Lafont, and I. J. Ortiz, Lower algebraic $K$-theory of hyperbolic 3-simplex reflection groups. Comment. Math. Helv. 84 (2009), 297-337. 
[L05] W. Lück, $K$ - and $L$-theory of the semi-direct product of the discrete 3-dimensional Heisenberg group by $\mathbb{Z} / 4$. Geom. Topol. 9 (2005), 1639-1676 Zbl 1073.19004 MR 2175154

[Mi71] J. Milnor, Introduction to algebraic K-theory. Ann. of Math. Stud. 72, Princeton University Press, Princeton, N.J., 1971. Zbl 0237.18005 MR 0349811

[St80] J. Stienstra, On $K_{2}$ and $K_{3}$ of truncated polynomial rings. In Algebraic $K$-theory, Evanston 1980, Lecture Notes in Math. 854, Springer-Verlag, Berlin 1981, 409-455. Zbl 0463.13007 MR 0618315

[vdK71] W. van der Kallen, Le $K_{2}$ des nombres duaux. C. R. Acad. Sci. Paris Sér. A-B 273 (1971), 1204-1207. Zbl 0225.13006 MR 0291158

[We80] C. A. Weibel, Mayer-Vietoris sequences and module structures on $N K_{*}$. In Algebraic K-theory, Evanston 1980, Lecture Notes in Math. 854, Springer-Verlag, Berlin 1981, 466-493. Zbl 0487.18012 MR 0618317

Received February 17, 2008

Charles Weibel, Department of Mathematics, Rutgers University, Piscataway, NJ 08854, U.S.A.

E-mail: weibel@math.rutgers.edu 